\title{
Long Term Non Linear Thermal Effects in the Pulsation of Mira Variables
}

\author{
A. Yaari and Y. Tuchman \\ Racah Institute, Hebrew University of Jerusalem, Jerusalem 91904, \\ Israel
}

The main conclusions that can be drawn from most of previous non-linear simulations of LPV (Keeley 1970, Rose \& Smith 1972, Wood 1974, Tuchman et al. 1978, 1979) are the following:

In all cases where the fundamental mode is predicted, by the linear analysis, to be the dominant, the pulsations are seen to diverge and lead to a rather violent mass-loss process (Wood 1974, Tuchman, Sack \& Barkat 1979).

A steady pulsation with (roughly) a limit cycle does set in only in those cases where the linear analysis predicts that the dominant mode (with the largest value of the growth rate) is the first overtone (or higher). In all these cases the actual limit cycle is found to be in a good agreement with the linear predictions, with regard to the pulsation mode and period (Wood 1974, Tuchman, Sack \& Barkat 1979). However, it is important to note that periods, for these overtone pulsators, are always found to be smaller than 300 days (Wood 1974, Tuchman, Sack \& Barkat 1979). Increasing the luminosity of these models, in order to increase their periods, results in a switch to the fundamental mode, followed by the violent relaxation oscillations, mentioned above.

The problem is that observed LPVs (Miras) exhibit a relatively clear periodic pulsation where most have periods between 300 and 400 days (Wood \& Cahn 1977). "Simple" modifications of the theoretical models, such as varying the mixing length, altering surface conditions, etc., are not found to help resolve the difficulty.

An additional problem of these first overtone pulsators is associated with their velocity amplitude which is found to be small compared to the observed values Hill \& Willson (1979).

In all previous works, calculating nonlinear dynamics of Miras, including our own, there was no clear identification that the star has indeed reached its limit cycle. To our surprise, it turned out, after continuing earlier calculations much farther (from about 50 years to more than 600 years!, Figure 1), that the non-linearity in the pulsation of a LPV causes a significant change of its internal energy structure (i.e., the entropy profile, see Figure 2). This change is accomplished in few hundred years $(\sim 300$ years which is about the thermal time scale of the envelope). As a new stable modified entropy profile is reached, a "true" limit cycle is seen to set in and is rather different from the earlier (obtained for $\sim 50$ years) "false" limit cycle (Figure 1). Pulsation has shifted from first overtone ( $\sim 175$ days) to a new fundamental mode ( $\sim 330$ days, no nodes) whose period is now between the first overtone and fundamental mode ( $\sim 508$ days) predicted by the linear analysis of the initial equilibrium model. 

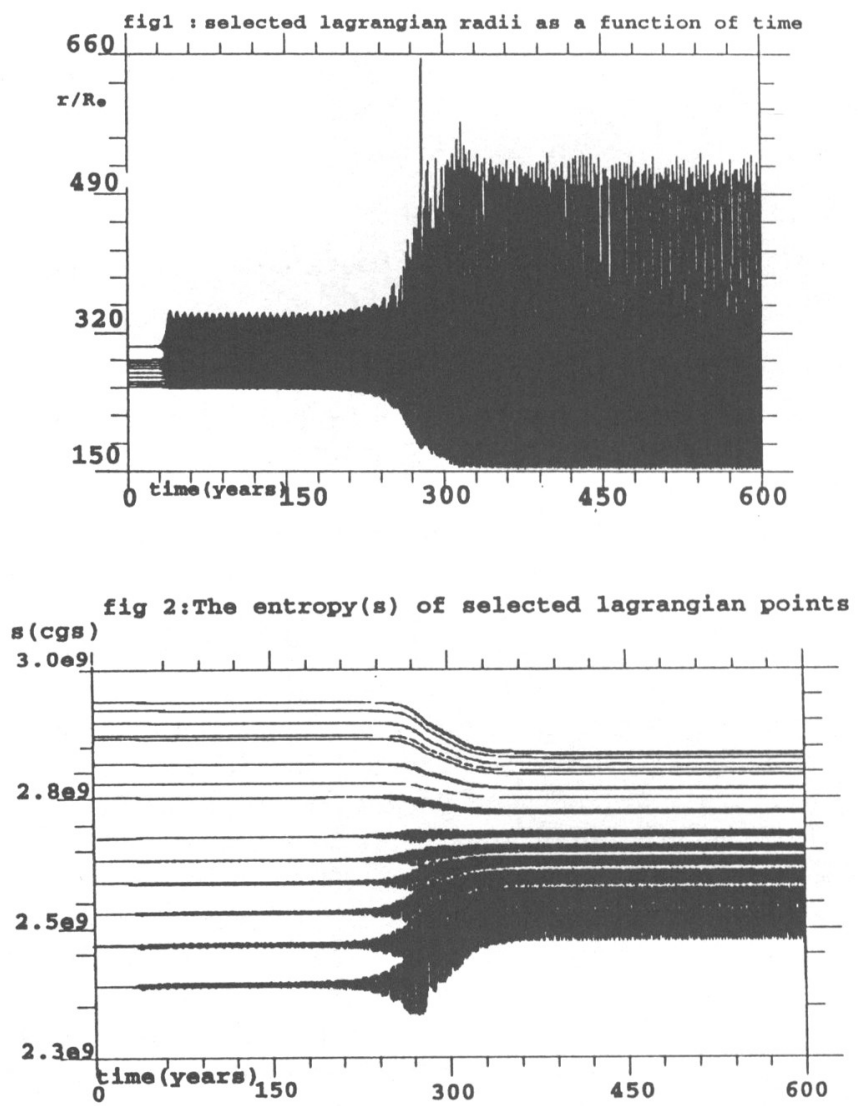

The pulsation features - periods, luminosity and velocity amplitudes - of these new nonlinear oscillating models are actually found to solve the two difficulties described above and to fit nicely the observational data.

The most important conclusion of these new results is that it is not possible to use the simple linear analysis for static models of Miras or Long Period Variables in order to determine their oscillatory behaviour, even their periods, as was and still is usually done.

\section{References}

Hill, S.A., \& Willson, L.A., 1979, ApJ, 229, 1029

Keeley, D.A., 1970, ApJ, 161, 656

Rose, W.K., \& Smith, R.L., 1970, ApJ, 159, 903

Tuchman, Y., Sack, N., \& Barkat, Z., 1978, ApJ, 219, 183

Tuchman, Y., Sack, N., \& Barkat, Z., 1979, ApJ, 234, 217

Wood, P.R., 1974, ApJ, 190, 609

Wood, P.R., \& Cahn, J.H., 1977, ApJ, 211, 499 